\title{
Children and adolescents with severe traumatic brain injury had verbal encoding deficits at 1 and 3 months after the injury
}

\author{
Roman MJ, Delis DC, Willerman L, et al. Impact of pediatric traumatic brain injury on components of verbal memory.J Clin Exp \\ Neuropsychol 1998 Apr;20:245-58.
}

\section{Question}

In children with traumatic brain injury (TBI), are components of verbal memory impaired several months after the injury?

\section{Design}

Inception cohort followed up for 3 months after the injury.

\section{Setting}

Inpatient trauma services of 3 San Diego (USA) area hospitals.

\section{Patients}

62 consecutively admitted children and adolescents between 6 and 16 years of age, 44 with TBI ( 17 with severe TBI and 27 with mild to moderate TBI) and 18 with other traumatic injuries (control group). Other inclusion criteria were right handedness; monolingual speakers of English; and no history of neurological difficulties, psychiatric illness, severe medical illness, mental retardation, substance abuse, previous head injury, or physical abuse. Children with a history of verbal learning disabilities were excluded, as were 10 patients unable to complete 2 evaluations.

\section{Assessment of prognostic factors}

At baseline, TBI victims were assigned to either the mildly to moderately injured or severely injured group based on post-resuscitation Glasgow Coma Scale scores. Traumatically injured patients without TBI were included to control for the emotional effects of trauma and admission to hospital. Demographic and treatment related information was collected at baseline.

\section{Main outcome measures}

Spared and impaired components of verbal learning and memory evaluated at 1 and 3 months after injury using the California Verbal Learning Test for Children (CVLT-C).

\section{Main results}

At 1 month, children with severe TBI had deficits in verbal memory task performance, which were characterised by impaired recall across 5 learning trials and after short and long delays. These patients also showed difficulties on recognition testing, with raised false positive rates among older patients, and deficient recognition hits among both older and younger patients. The degree of deficit in this patient group was generally mild with patients' mean scores falling between 0.5-1.0 SD below the normative mean. However, several patients with severe TBI had more substantial impairment with scores $>1.65 \mathrm{SD}$ below the normative mean (below the 5 th centile). Patients with severe TBI had a similar but less impaired profile at 3 months follow up. At 1 month and 3 months of follow up, patients with mild to moderate TBI did similarly to the control patients with both groups doing better than patients with severe TBI $(\mathrm{p}<0.05$ on most comparisons).

\section{Conclusion}

Children and adolescents with severe traumatic brain injury had verbal encoding deficits at 1 and 3 months after the injury.

Source of funding: not stated.

For correspondence: Dr D C Delis, University of California, San Diego, School of Medicine, Department of Psychiatry, Room 2072 Basic Science Building, 9500 Gilman Drive, La Jolla, California 92093-0603, USA. Fax +1 6195347653.

\section{Commentary}

This study by Roman et al is an excellent methodological example for outcome research in paediatric TBI. In addition to a non-biased sampling strategy, the characterisation of the patients according to injury severity and the inclusion of orthopaedic controls are exemplary. The findings are consistent with other studies showing that deficits in verbal learning are common sequelae of severe TBI. ${ }^{1}$ The results also support previous studies in showing that mild TBI is not associated with substantial long term sequelae in verbal learning and other domains of cognitive and behavioural functioning when premorbid difficulties are excluded. $^{2}$

Although it is reasonable to use the entire Glasgow Coma Scale (GCS) to characterise the severity of TBI, the most adverse outcomes are associated with 24 hour GCS motor scores $\leqslant 8$. This indicates that loss of consciousness for a day is a very good clinical indicator for progno- sis in the cognitive and behavioural domains. This indicator functions well in predicting a range of outcomes, all of which may be affected by severe TBI. ${ }^{3}$ Other studies have shown persistent deficits several years after the injury in these areas, including verbal learning. ${ }^{4}$

In addition to duration of unconsciousness, magnetic resonance imaging (MRI) studies in the 1-3 month interval after the injury also predict outcomes, particularly if there is evidence of frontal lobe damage. ${ }^{5}$ Future outcome studies would do well to incorporate MRI, particularly because it may predict outcome variation in the moderate injury group and because such studies may begin to point towards a mechanism. Other studies have found that performance on the CVLT-C is strongly related to the size of frontal lobe lesions. $^{5}$ In this study it is not clear whether children with severe TBI have difficulty with the CVLT-C because of the learning component, the emphasis on organisational strategies, or problems sustaining attention. More analytical studies in which performance on tasks like the CVLT-C is fractionated and then related to MRI findings, injury severity, and sociodemographic variables may help to explain the heterogeneity in the nature and severity of outcomes commonly associated with paediatric TBI.

Jack M Fletcher, $\mathrm{PhD}$ University of Texas - Houston Medical School Houston, Texas, USA

1 Fletcher JM, Taylor HG. Children with brain injury. In: Mash EJ, Terdal LG, editors. Assessment of childhood disorders. New York: Guilford Publications, 1997;453-80.

2 Satz P, Zaucha K, McCleary C, et al. Psychol Bull 1997;122:107-31.

3 Ewing-Cobbs LC, Duhaime AC, Fletcher JM. Journal of Head Trauma Rehabilitation 1995;10: 13-24.

4 Ewing-Cobbs L, Levin HS, Fletcher JM. In: Ylvisaker M, editor. Traumatic brain injury rehabilitation: children and adolescents. Boston: Buttersworth-Heinemann, 1998;11-26.

5 Levin HS, Culhane KA, Mendelsohn D, et al. Levin HS, Culhane KA, Mend
Arch Neurol 1993;50:897-905. 